\title{
Image Acquistion and Processing system based on USB
}

$$
\text { ZhangHui }^{1, \mathrm{a}} \quad \text { SiMing } \mathrm{He}^{1, \mathrm{a}} \quad \text { Changli Qiu, }{ }^{1, \mathrm{a}}
$$

${ }_{1,2,3,4,5}$ Electrical and Electronic Teaching and Research Section ,Aviation University of Air Force

Changchun, 130022, China

aemail:95832710@163.com

\section{Keywords: LabVIEW IMAQ USB USB camema}

Abstract. We use USB camera and IMAQ vision to program on LabVIEW platform. We process the contionuous acquistion of image,include adjusting the brightness, contrast, gamma value of image, and cut the image with a specified path.the design is low cost,easier implementation and flexible operation ,It's have good prospects for development.

\section{Introduction}

Image can help human perception to the outside world, is an important tool to obtain information,especially the video image is very convenience foe people's life.With the popularization and development of usb interface ,usb camera is widely used as high speed,portable image acquisition equipment.However, image acquistion process has some shortcoming such as long development cycle,poor universality, higher cost and so on .So ,we need design a strong commonality and hign performance of image and processing system is very important..In order to meet the above requirements, the design adopts LabVIEW as the software editor,which mainly used for data collection, analysis, display and control and other field.

We program with graphical programming language LabVIEW,through IMAQ USB module and USB camera to finish real-time acquisition and processing,system structure diagram is shown in Fig.1.The design is divided into two parts, the first part is including a computer equipped with LabVIEW sofyware,mainly for video image display,processing and preservation,the second part in cluding a common USB camera,focus on real-time image acquistion ${ }^{[1]}$.

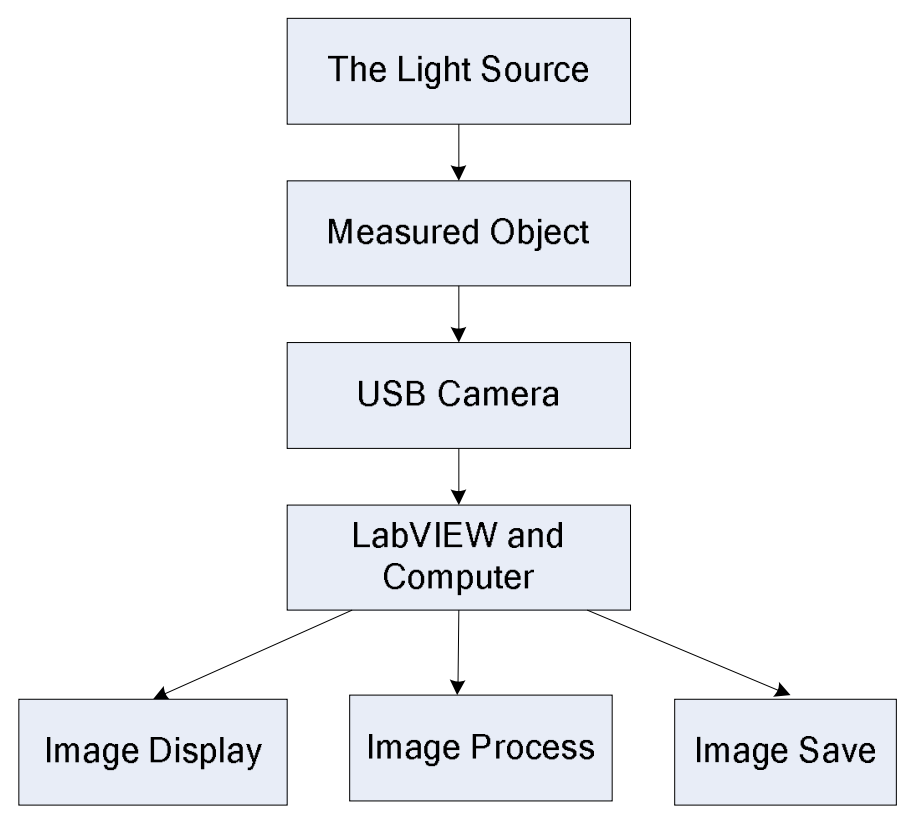

Fig.1. system structure diagram

For image acquisition and processing technology of labview platform,NI company provides powerfl software IMAQ VISION, which contains more than 400 image processing function and interactive image processing window, include statistics, filtering, image processing, such as geometric 
transformation shape matching,spot analysis and calulation and measurement application ${ }^{[2]}$.It can process one-dimensional or multidimensional image and also customize according to the requirement of design personalized features.

We use USB module of IMAQ VISION and USB camera to realize the video acquisttion,sedign control image operation panel,adjust the brightness of the image,contrast the gamma value ,capture image at a certain moment and save the specified durectory.the System is simple. The cycle is short, andoperation is flexible.

\section{The System Design Process}

The front panel Process. The front panel as the labview software user interface,can effectively collect video image and display on the computer.We can adjust brightness ,contrast and gamma value, at the same time, we can capture the image and save it under the specified path. The front panel design is shown in Fig.2.

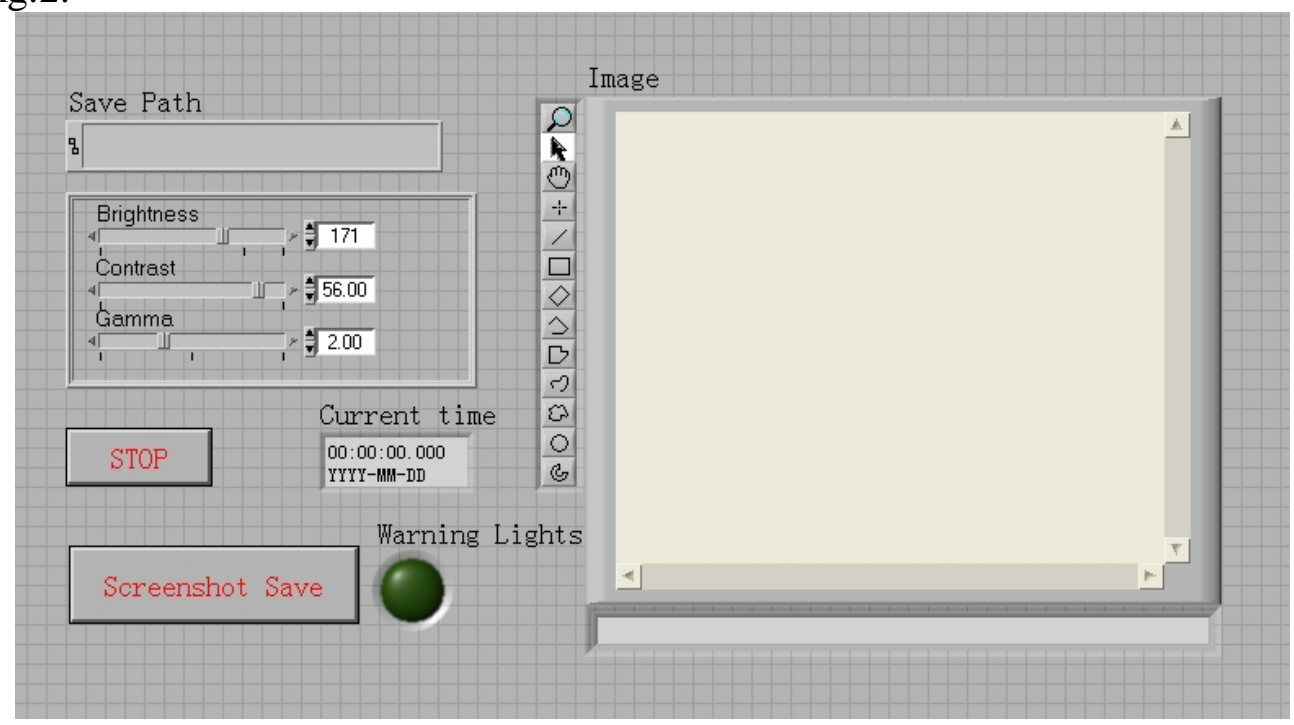

Fig.2. The front panel design

The diagram Design. The program block diagram is used to implement vi logic functions, is the graphical code,as shown in Fig.3.We use attachment to corresponding function such as program,controls, constants and variables.It's compared with the traditional text type code,not only the development is simple, but also the program is easy to understand ${ }^{[3]}$.

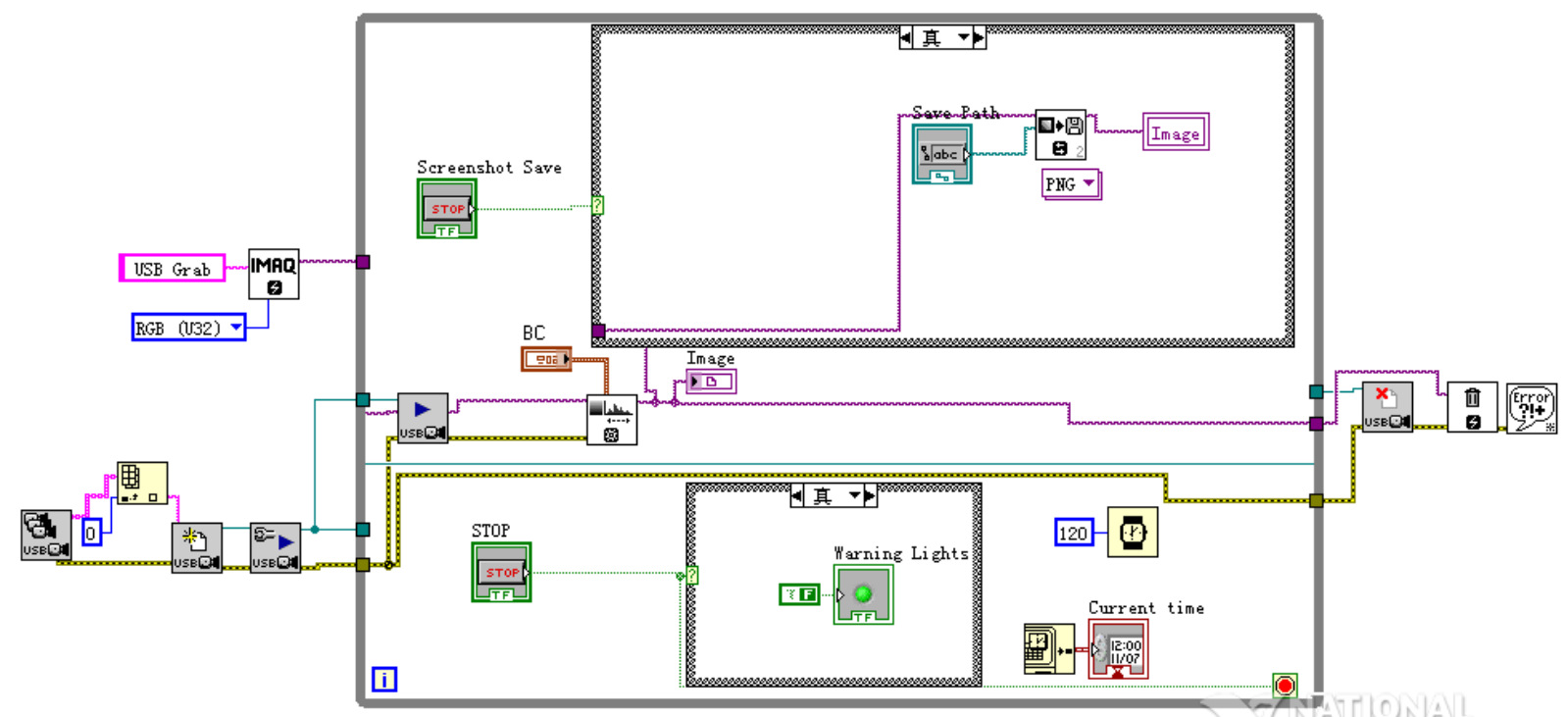

Fig.3. Theprogram panel design 
The program block diagram of the process as shown in Fig.4.the program block is divided into four main function moduled,including image acquistion module,image processing module,image saving module and other functionnal modules ${ }^{[4]}$.

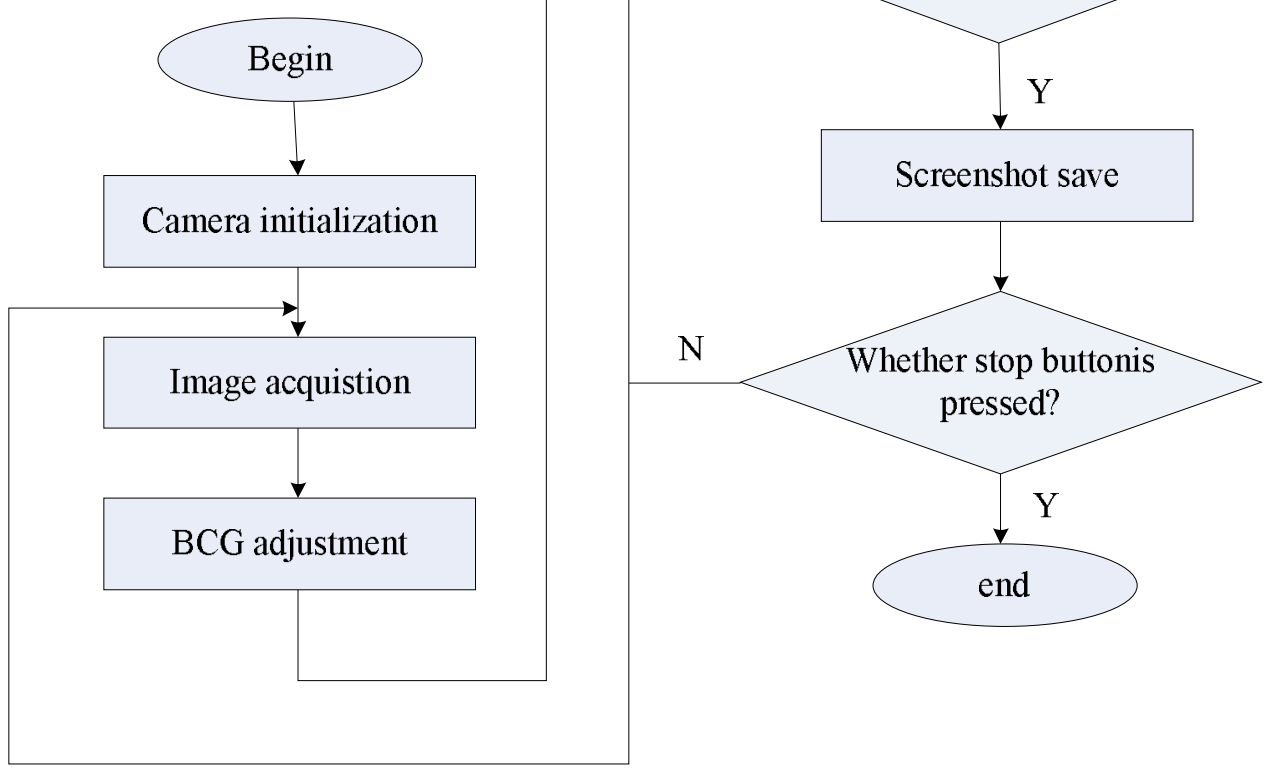

Fig.4 The program block diagram

(1)Image acquistion module:Firstly, we use the output of the IMAQ USB Enumer-ate Cameras.vi connected to the strings array of usb camera,open the USB camera with IMAQ USB Init.vi,and by IMAQ USB Init.vi to open the usb camera. Secondly,we use while loop and IMAQ USB Grab Acquire.vi to collet the image continnuous, and IMAQ Creat can set the buffer space of IMAQ USB Grab Acquire.vi.Finally, we use IMAQ USB Close.vi to close the USB camera. The whole process, the image real-time display by Image Dis-play.

(2)The Image processing module:We use the while loop to acquist the continuous image. We use IMAQ ColorB-CGLookup, Image Scr and Image Dst Out to connect input image and output image. R ed BCG Values, GreenBCG Values and Blue BCG Values are used to change the brightness,contrast and gamma value, which can adjust the visual effect of image.

(3)The save function:In the while loop,the conditional structure is used to judge whether we need screenshot.We use IMAQ WriteFile to set the save image type to PNG format,.The users can choose the srorage paths,including the select folder or new folder.Boolean controls the choice of control condition, if ture, the system automatically pop-up srore dialog box,you can customize the image name, and save the current video image; if false, wo must continues to collect images.In addition, if you select the cancel button in the dialog box,the system immediately to exit the save box,restore image acquisition function, does not affect the program run.

(4) Other modules: In the while loop,we can set the program to display the current time and ms timer.It is convenient for users to observation time by the current time mode. We can control code execution speed and reduce the CPU usage by ms timer.In addition, as long as the program runs,the red linghe has been bright, which can remind the user the program is using.

Image process. Fiestly, we process the image such as gradation transformation and smoothing filter. Secondly, we separate the target from the background.Thirdly, we product template and match template image.Finally, we calculate the center of the target location ${ }^{[5]}$.

Dynamic image recognition. The static image has nothing to do with the time, it is the function of the position,but only a static image can't descript the movement of objects. The moving target is image 
sequence, each image is called a frame, The different image contains of relative information of camera and scenery. The image sequence uaually can be expressed as $f(x, y, t)$,It add a time parameter $t$ compared with static image..Because of time interval of all imge is equal, so rhe image sequence can be expressed as $f(x, y, i)$,It means the number of frame. We can analysis image sequence and get the motion parameters.we can use a algorithm which compare two different frame of the target image and we can know the differences because of the movement of objects. In the actual calculation process, we need subtracte the two frame target image, according to difference pixel can detect the moving object, calculate the movement of the target direction.

\section{Conclusions}

Compared with traditional USB camera to collect video image, the system can not only realize the basic functions, abd when the image is darker,it is can adjust the brightness; the image is not clear,it is can adjust the contrast; $\gamma$ valuealso can help to adjust the image color information,improve the image quality,making it easy for users to observe.At the same time, the system has good development and application scenarios.

\section{References}

[1].P. Fornaro, M. Guggisberg, T. Gyalog, Ch. Wattinger, E. Meyer and H.-J. Gntherodt. A Remote Controllable and Programmable Atomic Force Microscope based on LabVIEW.(2008)

[2]. George C Panayi, Alan C Bovik and Umesh Rajashekar.Image Processing for Everyone.(2010)

[3]National Instruments. 《IMAQ Vision User's Manual》.(2011).

[4]Wang Zhixiao,Dong Fuguo.Realization of Virtual Oscillograph Based on USB.The Eighth International Conference on Electronic Measurement and Instruments,Xi' an,2007

[5]LabVIEW Advanced performance\&Communication Course Manual[J].National instruments,2013. 